\title{
Plasma nitrotyrosine in reversible myocardial ischaemia
}

\section{A Elfatih, N R Anderson, S Mansoor, S Ahmed, R Horton, M R Holland, R Gama}

Background: Nitric oxide (NO) plays a vital role in vascular homeostasis and in the pathophysiology of coronary heart disease. Its metabolites, nitrite and nitrate, have vasculoprotective properties, whereas peroxynitrite, an oxidant metabolite of NO, is cytotoxic and can aggravate myocardial damage during ischaemic reperfusion injury. Peroxynitrite nitrates free and protein bound tyrosine residues to produce nitrotyrosine. The measurement of nitrotyrosine provides an indirect estimation of plasma peroxynitrite concentrations.

Aims: To measure plasma nitrotyrosine concentrations to see whether peroxynitrite could contribute to myocardial dysfunction during myocardial ischaemia induced by an exercise tolerance test (ETT).

Materials/Methods: Plasma free nitrotyrosine concentrations were compared before and after exercise in 29 subjects with a positive ETT and 34 subjects with a negative ETT.

Results: Plasma nitrotyrosine concentrations were similar in patients with exercise induced myocardial ischaemia and controls.

Conclusion: Peroxynitrite does not contribute to the myocardial dysfunction in reversible myocardial ischaemia.

$\mathrm{N}$ itric oxide is released by the vascular endothelium and plays an important role in the maintenance of normal vascular endothelial function, cardiac function, and perfusion. $^{12}$ Peroxynitrite is a highly reactive oxidant metabolite of nitric oxide (NO). It is capable of inducing vascular cytotoxicity and myocardial dysfunction. ${ }^{3}$ It is generated in the vasculature from the reaction of NO with superoxide anions when both are present in micromolar concentrations under inflammatory and oxidative stress conditions associated with cytokine stimulated NO release. ${ }^{3}$ Peroxynitrite is widely recognised as a main mediator of NO toxicity associated with ischaemic perfusion injury, atherosclerosis, lung injury, and acute respiratory syndrome. ${ }^{34}$ Peroxynitrite nitrates free and protein bound tyrosine residues to yield nitrotyrosine, which is a stable end product used as a measure of circulating peroxynitrite concentration. ${ }^{5}$ Plasma nitrotyrosine has not been previously measured in the peripheral circulation during symptomatic myocardial ischaemia. Therefore, we compared plasma free nitrotyrosine concentrations before and after an exercise tolerance test (ETT) used to diagnose myocardial ischaemia.

\footnotetext{
"Peroxynitrite is widely recognised as a main mediator of nitric oxide toxicity associated with ischaemic perfusion injury, atherosclerosis, lung injury, and acute respiratory syndrome"
}

\section{SUBJECTS}

Subjects were recruited from a rapid access chest pain clinic. Exclusion criteria included recent acute myocardial infarction, unstable angina, cardiac surgery, uncontrolled hypertension, heart failure, claudication, transient ischaemic attack, stroke, and renal impairment. Subjects gave written consent to participate in our study, which was approved by the Wolverhampton district local research ethics committee.

\section{MATERIAL AND METHODS}

Venous blood was collected into lithium heparin tubes for the measurement of plasma free nitrotyrosine, 15 minutes before and 30 minutes after the ETT was performed according to the standard Bruce protocol. ${ }^{6}$ Samples were centrifuged within 30 minutes and the separated plasma was stored frozen at $-80^{\circ} \mathrm{C}$ until analysis. Plasma free nitrotyrosine was measured using a high performance liquid chromatography method described previously by Kaur et al, ${ }^{7}$ on a Gilson system with ultraviolet detection (Gilson SA, Villiers-Le-Bel, France). The assay has a detection limit of $0.20 \mu \mathrm{mol} /$ litre. The intra-assay coefficients of variation were $14 \%$ and $9.6 \%$ at $0.20 \mu \mathrm{mol} /$ litre and $1.0 \mu \mathrm{mol} /$ litre, respectively.

Data are expressed as median and range. The Mann Whitney $U$ test and the Wilcoxon matched pairs test were used to compare between and within group variables, respectively, using GraphPad, InStat computer software (GraphPad Software; San Diego, California, USA).

\section{RESULTS}

Table 1 shows the demographics of the 29 subjects with a positive ETT and the 34 subjects with a negative ETT. All patients with a positive ETT developed chest pain during exercise. Table $\mathrm{l}$ also shows the plasma nitrotyrosine concentrations before and after the ETT.

In summary, plasma nitrotyrosine concentrations before and after exercise were similar in subjects with a positive ETT and controls. Within each group, plasma nitrotyrosine was unaffected by exercise.

\section{DISCUSSION}

We report similar plasma nitrotyrosine concentrations in subjects with electrocardiographic myocardial ischaemia and those without myocardial ischaemia. Plasma nitrotyrosine has been shown to be generated under oxidative stress conditions with a severe endothelial insult, such as in chronic renal failure with septic shock, ${ }^{8}$ and in myocardial ischaemic reperfusion injury. ${ }^{9}$ However, our results suggest that moderate and reversible myocardial ischaemia, such as that induced by exertion, is not associated with an increased plasma peroxynitrite concentration. The generation of peroxynitrite requires increased circulating $\mathrm{NO}$ and superoxide radicals. ${ }^{3}$

Although raised circulating NO metabolites (nitrates) have been reported previously in patients with ischaemic heart disease compared with controls, ${ }^{10}{ }^{11}$ our study has shown that reversible myocardial ischaemia in the absence of myocardial damage does not generate sufficient superoxide anions to

Abbreviations: $E T T$, exercise tolerance test, $N O$, nitric oxide 
Table 1 Clinical and biochemical characteristics in subjects with a positive and negative exercise tolerance test

\begin{tabular}{llll}
\hline & Positive ETT & Negative ETT & p Value \\
\hline Number of subjects & 29 & 34 & \\
Number of men (\%) & $21(72.4 \%)$ & $19(55 \%)$ & \\
Age (years) & $64(39-79)$ & $52(31-68)$ & 0.0001 \\
Nitrotyrosine pre-ETT ( $\mu \mathrm{mol} / \mathrm{I})$ & $0.20(<0.2-1.2)$ & $0.20(<0.2-1.3)$ & 0.3125 \\
Nitrotyrosine post-ETT ( $\mu$ mol/l) & $0.20(<0.2-4.2)$ & $0.20(<0.2-1.6)$ & 0.084 \\
Time exercised (minutes) & $6.0(2-10)$ & $9.0(1-12)$ & 0.0001 \\
Maximum heart rate achieved & $85 \%(59-108 \%)$ & $100 \%(78-130 \%)$ & 0.0001 \\
\hline Values are given as median (range). & & & \\
ETT, exercise tolerance test. & & & \\
\end{tabular}

\section{Take home messages}

- We found that plasma nitrotyrosine concentrations before and after exercise were similar in subjects with myocardial ischaemia and controls

- Thus, peroxynitrite does not contribute to the myocardial dysfunction in exercise induced reversible myocardial ischaemia

overcome the scavenging role of superoxide dismutase and lead to increased generation of peroxynitrite.

In conclusion, plasma nitrotyrosine concentrations before and after exercise are similar in subjects with myocardial ischaemia and controls. Therefore, peroxynitrite does not contribute to the myocardial dysfunction in exercise induced reversible myocardial ischaemia.

\section{Authors' affiliations}

A Elfatih, N R Anderson, M R Holland, R Gama, Department of Clinical Chemistry, New Cross Hospital, Wolverhampton, West Midlands WV10 OQP, UK

S Mansoor, S Ahmed, R Horton, Departments of Cardiology, New Cross Hospital
Correspondence to: Dr A Elfatih, Department of Clinical Chemistry, New Cross Hospital, Wolverhampton, West Midlands WV10 OQP, UK; Abubaker.Elfatih@rwh-tr.nhs.uk

Accepted for publication 1 May 2004

\section{REFERENCES}

1 Ignarro L. Nitric oxide as a unique signalling molecule in the vascular system: a historical review. J Physiol Pharmacol 2002;53:503-14.

2 Vallance $P$, Hingorani A. Endothelial nitric oxide in health and disease. Int J Exp Pathol 1999;80:291-311.

3 Johansen JV. Physiological effects of peroxynitrite. Circ Res 2000;87:170-6.

4 Szabo C. The pathophysiological role of peroxynitrite in shock, inflammation and ischaemic-reperfusion injury. Shock 1996;6:79-88.

5 Claudia HP, Salesh K, Shuker EG. Analytical methods for nitrotyrosine as a marker of exposure to reactive nitrogen species. A review. Nitric Oxide Biology and Chemistry 1998;2:324-33.

6 Hill J, Timmis A. ABC of clinical electrocardiography. Exercise tolerance testing. Clinical review. BMJ 2002;324:1084-7.

7 Kaur H, Halliwell B. Evidence for nitric oxide mediated oxidative damage in chronic inflammation. Nitrotyrosine in serum and synovial fluid from rheumatoid patients. FEBS Lett 1994;350:9-12.

8 Fukuyama N, Takebayashi Y, Hida M, et al. Clinical evidence of peroxynitrite formation in chronic renal failure patients with septic shock. Free Radic Biol Med 1997;22:771-4.

9 Zweier JL, Fertmann J, Wei G. Nitric oxide and peroxynitrite in postischaemic myocardium. Antioxid Redox Signal 2001;3:11-22

10 Akarasereenont $P$, Nuamchit T, Khunawat P. Serum nitric oxide in patients with coronary artery disease. J Med Assoc Thai 2001;84:730-9.

11 Elfatih A, Anderson N, Mansoor S, et al. An investigation of nitric oxide metabolites during symptomatic myocardial ischaemia in relation to exercise tolerance test. Med Sci Monit 2003;9:CR511-14. 